Rev. Téc. Ing. Univ. Zulia. Vol. 44, No. 1, Enero-Abril, 2021, 12-20

\title{
Propiedades ópticas y mecánicas de biopelículas activas elaboradas con micropartículas de citral
}

\author{
Ives Yoplac ${ }^{1,2}$ (D) Patricio Córdova ${ }^{3}$ (D) Luis Vargas $^{4}$ (D) \\ ${ }^{1}$ Facultad de Ingeniería Zootecnista, Agronegocios y Biotecnología, Universidad Nacional Toribio Rodríguez de \\ Mendoza de Amazonas. Chachapoyas, Perú. \\ ${ }^{2}$ Escuela de Postgrado, Programa Doctoral en Ciencia de Alimentos, Universidad Nacional Agraria La Molina. \\ Lima, Perú. \\ ${ }^{3}$ Facultad de Educación y Ciencias de la Comunicación, Universidad Nacional Toribio Rodríguez de Mendoza de \\ Amazonas. Chachapoyas, Perú. \\ ${ }^{4}$ Facultad de Industrias Alimentarias, Universidad Nacional Agraria La Molina. Lima, Perú. \\ *Autor de Correspondencia: ives.yoplac@untrm.edu.pe \\ https://doi.org/10.22209/rt.v44n1a02
}

Recepción: 06 de enero de 2020 | Aceptación: 02 de octubre de 2020 | Publicación: 01 de enero de 2021

\section{Resumen}

Se estudió el efecto de la relación caseinato de sodio: sorbitol (CS:Sb) y la relación CS: micropartícula de citral (CS:MC), en las propiedades ópticas y mecánicas de biopelículas activas. Se siguió la aplicación de un arreglo factorial 3x3 con tres relaciones de CS:Sb (1:0,5; 1:1,0 y 1:1,5) y tres relaciones de CS:MC (1:0,5; 1:1,0 y 1:1,5). Se elaboraron mediante el método de casting, vertiendo la solución de formulación en placas de Petri y secando 24 horas a $25,0 \pm 0,5^{\circ} \mathrm{C}$ y $55,0 \pm 2,0 \%$ de humedad relativa. Se evaluaron las propiedades ópticas y mecánicas. Todas las variables evaluadas mostraron diferencias significativas en la interacción de factores y entre los niveles de factores. Se obtuvieron biopelículas entre 121 y $192 \mu \mathrm{m}$ de espesor. El incremento significativo del espesor tuvo relación directa con el aumento de las relaciones CS:Sb y CS:MC. Se observó que los mejores resultados, con respecto a las propiedades ópticas de las biopelículas activas, fueron encontrados para formulaciones con menores niveles de relación CS:Sb y CS:MC, obteniendo menor diferencia de color $(7,6)$, menor opacidad $(14,8 \%)$ y menor valor de transparencia $\left(1,4 \mathrm{~A}_{600} / \mathrm{mm}\right)$. Respecto a las propiedades mecánicas de las biopelículas, los mejores resultados se encontraron para formulaciones con altas concentraciones de sorbitol y bajas concentraciones de MC, observándose mayor deformación (63\%) y menor resistencia a la tracción (1,9 MPa).

Palabras clave: deformación; envase activo; módulo elástico; opacidad; valor de transparencia.

\section{Optical and mechanical properties of active biofilms made with citral microparticles}

\begin{abstract}
The effect of the sodium caseinate:sorbitol (CS:Sb) and CS:citral microparticle (CS:MC) ratio on the optical and mechanical properties of active biofilms was studied. The application of a $3 \times 33 \times 3$ factorial arrangement was followed with three CS:Sb ratios (1:0.5, 1:1.0 and 1:1.5) and three CS:MC ratios (1:0.5, 1:1.0 and 1:1.5). It was made by casting method, pouring the formulation solution into Petri dishes and dried 24 hours at $25 \pm 0.5^{\circ} \mathrm{C}$ and $55 \pm 2.0 \%$ relative humidity. Optical and mechanical properties were evaluated. The studied variables showed significant differences in the interaction of the factors and between the levels of the factors. Biofilms between 121 and $192 \mu$ m thick were obtained. The significant increase in thickness was directly related to higher CS:Sb and CS:MC ratios. It was observed that the best results regarding the active biofilms optical properties were found for formulations with lower levels of CS:Sb and CS:MC, obtaining less color difference (7.6), less opacity $(14.8 \%)$ and less transparency value $\left(1.4 \mathrm{~A}_{600} / \mathrm{mm}\right)$. Regarding the biofilms mechanical properties, the best results were found for formulations with high sorbitol concentrations and low MC concentrations, showing greater deformation (63\%) and less tensile strength (1.9 MPa).
\end{abstract}

Keywords: deformation; active package; elastic modulus; opacity; transparency value. 


\section{Introducción}

La industria del envase y embalaje es una de las más dinámicas en el mundo, por su importancia en las diversas cadenas de valor. Por ello, este sector alcanzó, en el 2018, una producción mundial de 975 mil millones de dólares [1].

Los envases y embalajes son ampliamente usados en diferentes sectores industriales, como la industria alimentaria. En este sector, los materiales de elaboración para envase primario más usados son papel, cartón, plásticos, metal y vidrio [2,3]. De todos estos, los envases de plástico han aumentado su participación en los últimos años, y ocupan el $45 \%$ del total del mercado de envases, debido a su peso ligero, versatilidad y bajo costo $[1,4]$. No obstante, los envases plásticos provienen de derivados del petróleo, que son fuentes no renovables, no biodegradables, tardan más de cien años para su degradación y, por ende, causantes de contaminación ambiental; siendo los principales el polietileno, poliestireno y polipropileno [5,6].

Frente a esta problemática, los biopolímeros son considerados como recursos biotecnológicos con propiedades únicas como la ausencia de toxicidad, degradación y compatibilidad biológica; además, se constituyen en fuente para el desarrollo de biopelículas biodegradables [3]. Los principales biopolímeros utilizados en este propósito son obtenidos a partir del almidón, celulosa, algas marinas, quitosano, escamas de pescados, fuentes proteicas, semillas de frutos, entre otros; a los que se le adiciona otros materiales como lípidos, plastificantes, agentes activos y solventes [7-10]. Dentro de los biopolímeros de fuentes proteicas, destacan los obtenidos de productos lácteos como la caseína $[11,12]$.

Las biopelículas de caseína son transparentes, biodegradables y presentan buenas propiedades de barrera al oxígeno; además, pueden usarse como soporte de compuestos antimicrobianos, o antioxidantes (biocompuestos), dándole a la biopelícula una propiedad funcional conocida como biopelícula activa o envase activo [13-15]. La adición de estos biocompuestos se potencia cuando se incorpora protegido, por ejemplo, mediante microencapsulación [16,17]. Estas biopelículas activas son de gran utilidad para el envasado de alimentos perecederos como lácteos, cárnicos, hortofrutícolas, alimentos preparados, entre otros. En algunos casos estudiados se muestran los siguientes beneficios: reducir la pérdida de agua, permeabilidad al oxígeno, retardar la oxidación de lípidos, mejorar la textura y el sabor, disminuir el recuento microbiano y en general prolongar la vida útil del alimento a partir de la interacción de los biocompuestos con el envase [8].

La elaboración de biopelículas activas con propiedades antimicrobianas y/o antioxidantes se puede formular con incorporación de sustancias naturales como extractos o agentes activos (compuestos o metabolitos secundarios) extraídos de los aceites esenciales (AE), en forma libre o microencapsulado [18-21]. De los agentes activos (AA), destacan el mentol, geraniol, timol, eugenol, carvacrol, citral, entre otros [22-25]. Por su lado, el citral (3, 7-Dimetil-2, 6-octadienal) es un aldehído monoterpeno acíclico, compuesto por dos isómeros geométricos: geranial (citral $A$ en su forma cis) y neral (citral $B$ en su forma trans) [26,27]. Este AA principalmente posee actividad antimicrobiana [28].

La literatura reporta algunos estudios donde incorporaron el citral microencapsulado en la formulación de biopelículas activas, entre el más reciente destaca los reportados por Alarcón-Moyano et al. [16], quienes adicionaron a una matriz de alginato de sodio, obteniendo biopelículas estables y reducción microbiana en ensayos in vitro. Por otro lado, existen estudios donde se emplearon caseinato de sodio en la elaboración de biopelículas activas con la incorporación de AA en forma libre, como carvacrol [14,15], AE de germen de maíz [29], AE de tung [30]. La mayoría de los trabajos antes mencionados coinciden en que la adición de los diferentes niveles del biopolímero, plastificante y agente activo influyen significativamente en las propiedades físicas, ópticas y mecánicas de la biopelícula. Sin embargo, no se encontró evidencia del uso de citral microencapsulado con caseinato de sodio y sorbitol para la elaboración de biopelículas activas.

Por lo tanto, el presente estudio tuvo como objetivo evaluar el efecto del caseinato de sodio, sorbitol y micropartículas de citral en las propiedades ópticas y mecánicas de biopelículas activas.

\section{Materiales y métodos}

\section{Reactivos}

Para la elaboración de la biopelícula activa se emplearon: caseinato de sodio, sorbitol (Sigma - Aldrich, Alemania) y micropartículas de citral (tamaño medio de $7,08 \mu \mathrm{m}$ ) obtenidas en investigaciones previas [31,32], elaboradas con citral y lecitina de soya (Sigma - Aldrich, Alemania) y dextrina (Ingredion, Perú).

\section{Preparación de las biopelículas activas}

Las biopelículas se prepararon siguiendo la metodología planteada por Arrieta et al. [15], con modificaciones. Las soluciones se formularon en agua destilada con $5 \% \mathrm{~m} / \mathrm{v}$ de caseinato de sodio (CS). Se añadió sorbitol $(\mathrm{Sb})$ para obtener proporciones de CS: $\mathrm{Sb}(1: 0,5 ; 1: 1,0$ y $1: 1,5)$. Se mezclaron a $50{ }^{\circ} \mathrm{C}$ durante 10 minutos bajo agitación continua a $1000 \mathrm{rpm}$ en un agitador magnético (Thermo Scientific, SP131015 Cimarec, USA), luego se enfriaron a temperatura ambiente. El pH medio de las soluciones de CS-Sb fue de 6,48 $\pm 0,01$. Posteriormente, se añadieron las micropartículas de citral 
(MC) en una proporción de CS: MC (1:0,5; 1:1,0 y 1:1,5), se mezcló a $35{ }^{\circ} \mathrm{C}$ durante 5 minutos a $1000 \mathrm{rpm}$. El pH medio de las soluciones finales fue de 6,39 $\pm 0,04$. Finalmente, a todas las soluciones se les realizo una desgasificación ultrasónica (QSonica, Q55, Newtown CT, USA) a 35\% de intensidad digital, durante 10 minutos y a temperatura ambiente, para eliminar las espumas y las burbujas de aire.

Las películas se obtuvieron mediante el método de casting, vertiendo $9 \mathrm{~mL}$ de estas soluciones en recipientes de placas de Petri de vidrio de $9 \mathrm{~cm}$ de diámetro (EULab, Alemania); obteniendo un total de 4 placas de Petri por solución (tratamiento, Tabla 1). Se acondicionaron durante 24 horas a $25 \pm 0,5{ }^{\circ} \mathrm{C}$ y $55 \pm 2,0 \%$ de humedad relativa (HR), en una cámara de secado con aire forzado Venticell-VC222 (MMM Group, Alemania), equipada con un higrómetro Traceable ${ }^{\circledR}$ (Thermo Scientific, USA). Las biopelículas activas resultantes fueron almacenadas a temperatura ambiente, dentro de bolsas de polietieleno debidamente rotuladas, para su posterior evaluación dentro de las primeras 48 horas; a excepción del valor de transparencia que se midió a los 14 días posteriores a su obtención, para evidenciar diferencias significativas entre los tratamientos.

\section{Evaluaciones de las biopelículas activas}

\section{a) Espesor}

El espesor medio de las películas se midió con un micrómetro Digimatic IP-65, serie 293-240 (Mitutoyo, Japón) con $\pm 0,001 \mathrm{~mm}$ de precisión, en cinco posiciones aleatorias sobre la superficie de tres biopelículas por tratamiento.

\section{b) Propiedades ópticas}

\section{- Color}

W La medición de color de las biopelículas se realizó según lo descrito por Pires et al. [33] y se aplico sobre la superficie de un respaldo blanco estándar $\left(L^{*}=\right.$ 93,$11 ; a^{*}=-0,63$ y $\left.b^{*}=3,82\right)$. Los parámetros de color $\left(L^{*}\right.$, $a^{*}$ y $b^{*}$ ) se midieron con un colorímetro CR-400 (Konica Minolta Co., Ltd., Osaka, Japón). Los valores de blancura $(W)$ de las biopelículas se calcularon usando la ecuación (1):

$$
W=100-\left(\left(100-L^{*}\right)^{2}+a^{* 2}+b^{* 2}\right)^{1 / 2}
$$

El color de las películas se expresó como la diferencia de color $\left(\Delta E^{*}\right)$ y se calculó con la ecuación (2) [33].

$$
\Delta E^{*}=\sqrt{\left(\Delta L^{*}\right)^{2}+\left(\Delta a^{*}\right)^{2}+\left(\Delta b^{*}\right)^{2}}
$$

donde $\Delta \mathrm{L}^{*}, \Delta \mathrm{a}^{*}, \Delta \mathrm{b}^{*}$ son los diferenciales entre el parámetro de color de las muestras y el parámetro de color de una cartulina blanca estándar utilizado como fondo o respaldo de la película. Se realizaron pruebas de color en cuatro posiciones diferentes de tres biopelículas por tratamiento.

\section{- Opacidad}

Para la medición de la opacidad se siguieron las indicaciones del método HunterLab [34] y la metodología propuesta por Pires et al. [33], con la utilización de un cromámetro CR-400 (Konica Minolta Co., Ltd., Osaka, Japón). La opacidad (\%) de las muestras se calculó con la ecuación (3), a partir de las mediciones de reflectancia de cada una con una base o fondo negro $\left(Y_{\text {Fondo negro }} L^{*}=21,84\right.$; $a^{*}=0,29$ y $\left.b^{*}=1,70\right)$ y una base o fondo blanco $(Y$ $L^{*}=93,12 ; a^{*}=-0,65$ y $\left.b^{*}=3,99\right)$.

$$
\text { Opacidad }=\frac{Y_{\text {Fondonegro }}}{Y_{\text {Fondoblanco }}} \times 100
$$

donde $Y$ es el valor triestímulo $Y$; considerando que el concepto de valores triestímulo (X, Y, Z), se basa en la teoría de los tres componentes de la visión del color, que establece que el ojo posee receptores para tres colores primarios (rojo, verde, azul) y que todos los colores se ven como mezclas de estos tres colores primarios. Estos valores triestímulos XYZ forman la base para definir el sistema de color CIELab* $[35,36]$. Se realizaron pruebas de opacidad en cuatro posiciones diferentes de tres biopelículas por tratamiento.

\section{- Transparencia}

La transparencia de las biopelículas se calculó mediante la ecuación (4), según lo informado por Hamaguchi et al. [37], Pires et al. [33] y Shiku et al. [38], mediante un espectrofotómetro Genesys 10S UVVIS (Thermo Fisher Scientific, EE.UU), para lo cual se cortaron tiras de 10x80 mm (ancho x largo) y se colocaron en cubetas de cuarzo para su medición. Como blanco se midieron cubetas vacías.

$$
\text { Valordetransparencia }=\frac{A_{600}}{x}
$$

donde $A$ es la absorbancia a $600 \mathrm{~nm}$ y $x$ es el espesor de la película (mm). De acuerdo con esta ecuación, los valores de transparencia más altos indican una menor transparencia. La transparencia se midió a los 14 días después del proceso de obtención, para evidenciar la diferencia entre tratamientos, debido a que en los primeros días no es notable; se realizó en tres biopelículas diferentes por tratamiento.

\section{c) Propiedades mecánicas}

\section{- Módulo elástico (E)}

Los ensayos de tracción se llevaron a cabo 
a temperatura ambiente y 50\% de HR, utilizando un texturómetro Instron Instrument 3365 (Fareham Hants, Reino Unido), según el estándar ASTM D882-01 [39] y la metodología propuesta por Arrieta et al. [15], con modificaciones. Las pruebas se realizaron en tiras rectangulares $(10 \times 80 \mathrm{~mm})$, separación de agarre inicial de $40 \mathrm{~mm}$ y velocidad de la cruceta de $25 \mathrm{~mm} / \mathrm{min}$. La media del módulo elástico $(E)$ medido en $\mathrm{MPa}$, se calculó a partir de las curvas de fuerza $(\mathrm{N})$-extensión $(\mathrm{mm})$ resultantes, como la media de tres biopelículas de cada tratamiento.

\section{- Alargamiento a la rotura $\left(\varepsilon_{\mathrm{B}}\right)$ y resistencia a la trac- ción (RT)}

El alargamiento a la rotura (deformación) y resistencia a la tracción fueron calculados en \% y $\mathrm{MPa}$, respectivamente, a partir de las curvas de fuerza (N)-extensión (mm) resultantes, como la media de tres biopelículas por tratamiento y de acuerdo a las metodologías propuestas por Arrieta et al. [15] y ASRM [39].

En el presente estudio, para la selección del mejor tratamiento, se tuvo en cuenta aquel que para las propiedades ópticas, presentara menor $\mathbf{\Delta E}$, opacidad y VT, así como, mayor porcentaje de blancura. Para propiedades mecánicas, aquel que presentara menor $E$ y RT, así como, mayor porcentaje de $\boldsymbol{\varepsilon}_{\mathrm{B}}$.

\section{Diseño experimental y análisis de datos}

En este estudio, se utilizó un diseño completamente al azar (DCA) con estructura factorial $3 \times 3$. Los factores evaluados correspondieron a la relación $\mathrm{CS}: \mathrm{Sb}$, con tres niveles $(1: 0,5 ; 1: 1,0$ y $1: 1,5)$ y relación CS:MC con tres niveles $(1: 0,5 ; 1: 1,0$ y $1: 1,5)$. De esta combinación, surgieron nueve tratamientos y cada uno con tres repeticiones (Tabla 1). La unidad experimental correspondió a una biopelícula activa de $9 \mathrm{~cm}$ de diámetro. Los residuos obtenidos a partir de los datos de las variables evaluadas (propiedades ópticas y mecánicas), se sometieron a una prueba de normalidad y homogeneidad de varianza. Luego de verificar los supuestos, se procedió a realizar un análisis de varianza (ANDEVA), con un 5\% de significancia.

Cuando se evidenció interacción significativa entre los factores (tratamientos), se aplicó una prueba de comparaciones de medias de Tukey $(\alpha \leq 5 \%)$ entre los tratamientos. Cuando solo existieron diferencias significativas en los niveles de por lo menos un factor, se realizó una prueba de comparación de medias de Tukey $(\alpha$ $\leq 5 \%$ ) entre los niveles de ese factor.

Los resultados fueron analizados estadísticamente mediante el programa Minitab Release 17,1; software estadístico de acceso libre para Windows.
Tabla 1. Distribución de los factores y tratamientos para la obtención de las biopelículas activas.

\begin{tabular}{ccc}
\hline $\begin{array}{c}\text { Factor A } \\
\text { Relación CS:Sb }\end{array}$ & $\begin{array}{c}\text { Factor B } \\
\text { Relación CS:MC }\end{array}$ & Tratamiento \\
\hline $1: 0,5$ & $1: 0,5$ & $\mathrm{~T} 1: 0,5+0,5$ \\
$1: 0,5$ & $1: 1,0$ & $\mathrm{~T} 2: 0,5+1,0$ \\
$1: 0,5$ & $1: 1,5$ & $\mathrm{~T} 3: 0,5+1,5$ \\
$1: 1,0$ & $1: 0,5$ & $\mathrm{~T} 4: 1,0+0,5$ \\
$1: 1,0$ & $1: 1,0$ & $\mathrm{~T} 5: 1,0+1,0$ \\
$1: 1,0$ & $1: 1,5$ & $\mathrm{~T} 6: 1,0+1,5$ \\
$1: 1,5$ & $1: 0,5$ & $\mathrm{~T} 7: 1,5+0,5$ \\
$1: 1,5$ & $1: 1,0$ & $\mathrm{~T} 8: 1,5+1,0$ \\
$1: 1,5$ & $1: 1,5$ & $\mathrm{~T} 9: 1,5+1,5$ \\
\hline
\end{tabular}

CS: caseinato de sodio; Sb: sorbitol; MC: micropartículas de citral.

\section{Resultados y discusión}

Los resultados para todas las variables evaluadas mostraron diferencias significativas en la interacción de los factores y en los niveles de los factores $(\mathrm{p}<0,05)$.

\section{Espesor}

La Tabla 2 muestra los valores del espesor de las biopelículas activas, que varió entre 121,3 y 192,0 $\mu \mathrm{m}, \mathrm{y}$ fueron superiores a los reportados por Arrieta et al. $[14,15]$, para biopelículas de CS con agente activo (AA) de carvacrol libre $(88 \pm 16 \mu \mathrm{m})$. Esto se debería, principalmente, a la incorporación, en el presente estudio, del AA de citral microencapsulado con dextrina y lecitina de soya, solutos que influyeron en el incremento del espesor.

Como se había previsto, se observó que la adición de solutos en la solución contribuyó con el incremento del espesor de las biopelículas. Se evidenció que las relaciones CS:Sb y CS:MC, ambos con nivel 1:0,5; produjeron biopelículas con menor espesor. Del mismo modo, se constató que T1 y T4, ambos con el menor contenido de plastificante y MC, exhibieron biopelículas más delgadas con 121,3 y 143,8 $\mu \mathrm{m}$, respectivamente. Se observó cambio significativo con la adición de plastificante y MC. Estos resultados son similares a los reportados por Pereda et al. [30] para biopelículas con CS y AE de tung, y que muestran una relación directa entre el espesor y el contenido de solutos en la formulación.

\section{Propiedades ópticas}

La blancura de las biopelículas activas varió de 73,1 a 85,4 (Tabla 3), siendo inferior a la reportada por Pires et al. [33], para biopelículas elaboradas con proteína 
Tabla 2. Espesor de las biopelículas activas en los tratamientos evaluados.

\begin{tabular}{ccccl}
\hline Tratamiento (T) & \multicolumn{5}{c}{ Espesor $(\boldsymbol{\mu m})$} \\
X $\pm \mathrm{DE}$ \\
\hline T1: $0,5+0,5$ & $121,27^{1}$ & \pm & 6,20 & $\mathrm{a}$ \\
T2: $0,5+1,0$ & 154,53 & \pm & 10,63 & $\mathrm{bc}$ \\
T3: $0,5+1,5$ & 159,47 & \pm & 1,21 & $\mathrm{bc}$ \\
T4: $1,0+0,5$ & 143,80 & \pm & 12,93 & $\mathrm{ab}$ \\
T5: $1,0+1,0$ & 164,78 & \pm & 3,38 & $\mathrm{bcd}$ \\
T6: $1,0+1,5$ & 170,73 & \pm & 22,15 & $\mathrm{bcd}$ \\
T7: $1,5+0,5$ & 179,07 & \pm & 1,75 & $\mathrm{~cd}$ \\
T8: $1,5+1,0$ & 189,93 & \pm & 0,99 & $\mathrm{~d}$ \\
T9: $1,5+1,5$ & 192,00 & \pm & 8,34 & $\mathrm{~d}$ \\
\hline
\end{tabular}

${ }^{1}$ Los valores indican la media $(n=3)$. X: media; DE: desviación estándar.

Letras diferentes, en sentido vertical, indican diferencias estadísticamente significativa según la prueba Tukey $(\mathrm{p}<0,05)$, para cada tratamiento $(\mathrm{T})$. de merluza y AE de tomillo. Las biopelículas con relacones CS:Sb de $(1: 0,5$ y 1:1,0) y CS:MC $(1: 1,0)$; es decir, T1 y $\mathrm{T} 4$, presentaron los mayores valores de blancura, siendo estos tratamientos los más transparentes. Por otro lado, al evaluar las interacciones se observó que T3, T6 y T9, todos con mayor contenido de MC, mostraron menores valores de blancura; resultados que demuestran que estos tratamientos fueron menos transparentes, debido principalmente al incremento de MC en la formulación para la obtención de las biopelículas activas. Estos resultados fueron diferentes a los obtenidos por Pires et al. [33], quienes observaron que las diferentes concentraciones de AE de tomillo, adicionado a la proteína de merluza, no influyó significativamente en la blancura de la biopelícula, debido probablemente a que fue añadido en forma libre. En el presente estudio, en cambio, el AA fue adicionado microencapsulado, donde además del citral, contenía dextrina y lecitina de soya (encapsulante y emulsifcante, respectivamente), solutos que por sus propias características bioquímicas, físicas y ópticas, podrían ser los responsables de valores bajos de blancura.

Tabla 3. Propiedades ópticas de las biopelículas activas en los tratamientos evaluados.

\begin{tabular}{|c|c|c|c|c|c|c|c|c|c|c|c|c|}
\hline \multirow{3}{*}{$\begin{array}{c}\text { Tratamiento (T) } \\
\text { T1: } 0,5+0,5\end{array}$} & \multicolumn{12}{|c|}{ Propiedades ópticas } \\
\hline & \multicolumn{3}{|c|}{$\underset{\mathrm{X} \pm \mathrm{DE}}{\text { Blancura }}$} & \multicolumn{3}{|c|}{$\begin{array}{l}\Delta \mathbf{E}^{* 2} \\
\mathrm{X} \pm \mathrm{E}\end{array}$} & \multicolumn{3}{|c|}{$\begin{array}{c}\text { Opacidad (\%) } \\
X \pm D E\end{array}$} & \multicolumn{3}{|c|}{$\begin{array}{c}\mathrm{VT}^{3} \text { - } 14 \text { días } \\
\left(\mathrm{A}_{600} / \mathbf{m m}\right) \\
\mathrm{X} \pm \mathrm{DE}\end{array}$} \\
\hline & $85,37^{1}$ & \pm & $0,56 \mathrm{f}$ & 7,61 & \pm & $0,58 \mathrm{a}$ & 14,82 & \pm & $0,90 \mathrm{a}$ & 2,40 & \pm & $0,40 \mathrm{ab}$ \\
\hline $\mathrm{T} 2: 0,5+1,0$ & 78,32 & \pm & $0,29 \mathrm{c}$ & 15,09 & \pm & $0,30 \mathrm{c}$ & 17,38 & \pm & $0,60 \mathrm{abc}$ & 1,36 & \pm & $0,42 \mathrm{a}$ \\
\hline T3: $0,5+1,5$ & 73,05 & \pm & $0,85 \mathrm{a}$ & 20,59 & \pm & $0,86 \mathrm{e}$ & 19,99 & \pm & $1,11 \mathrm{c}$ & 1,99 & \pm & $0,10 a b$ \\
\hline $\mathrm{T} 4: 1,0+0,5$ & 84,88 & \pm & 0,49 ef & 8,03 & \pm & $0,50 \mathrm{a}$ & 16,34 & \pm & $0,90 \mathrm{abc}$ & 5,80 & \pm & $1,14 \mathrm{c}$ \\
\hline T5: $1,0+1,0$ & 79,72 & \pm & $0,70 \mathrm{~cd}$ & 13,61 & \pm & $0,69 \mathrm{bc}$ & 15,71 & \pm & $0,31 \mathrm{ab}$ & 2,63 & \pm & $0,22 \mathrm{ab}$ \\
\hline T6: $1,0+1,5$ & 75,54 & \pm & $1,71 \mathrm{ab}$ & 18,11 & \pm & $1,68 \mathrm{de}$ & 16,20 & \pm & $0,90 \mathrm{abc}$ & 3,32 & \pm & $0,57 \mathrm{~b}$ \\
\hline $\mathrm{T} 7: 1,5+0,5$ & 82,20 & \pm & 0,68 de & 11,07 & \pm & $0,75 \mathrm{~b}$ & 33,66 & \pm & $2,57 \mathrm{e}$ & 8,29 & \pm & $0,69 \mathrm{~d}$ \\
\hline T8: $1,5+1,0$ & 77,26 & \pm & $1,06 \mathrm{bc}$ & 16,16 & \pm & $1,09 \mathrm{~cd}$ & 25,37 & \pm & $2,64 d$ & 7,11 & \pm & $0,80 \mathrm{~cd}$ \\
\hline T9: $1,5+1,5$ & 75,01 & \pm & $1,44 \mathrm{ab}$ & 18,64 & \pm & $1,25 \mathrm{de}$ & 19,21 & \pm & $0,11 \mathrm{bc}$ & 6,83 & \pm & $0,72 \mathrm{~cd}$ \\
\hline
\end{tabular}

${ }^{1}$ Los valores indican promedio $(\mathrm{n}=3)$. X: la media; $\mathrm{DE}$ : desviación estándar.

${ }^{2}$ Diferencia del color total.

${ }^{3}$ Valor de transparencia.

Letras diferentes en sentido vertical, indican diferencias estadísticamente significativas según la prueba Tukey $(\mathrm{p}<0,05)$, para cada tratamiento (T).

La $\Delta \mathrm{E}^{*}$ varió entre 7,6 y 20,6 (Tabla 3); valores superiores a los reportados por Pires et al. [33], para biopelículas con proteína de merluza y AE de tomillo. Al evaluar los niveles de los factores, se observó que la relación CS:Sb con nivel 1:1,0 y la relación CS:MC con nivel de 1:0,5; mostraron menores valores de $\Delta \mathrm{E}^{*}$. Al analizar las interacciones de los factores, se observó que los tratamientos T1 y T4, ambos con menor contenido de MC, presentaron los menores valores de $\Delta \mathrm{E}^{*}$.

La opacidad varió entre 14,8 y 33,7\% (Tabla 3), los cuales estan dentro de los rangos reportados por Pires 
et al. [33], para biopelículas con proteínas de merluza, glicerol y AE de tomillo (15 y 16\%). Por el contrario, fueron superiores a los seálados por García y Sobral [40] para biopelículas de proteína de tilapia y glicerol (4 y $10 \%$ ), siendo más transparentes que las biopelículas del presente estudio. Esto podría deberse principalmente a la adición de AA en la formulación, al igual que en otros estudios donde la opacidad de las biopelículas se vio influenciada principalmente por el AA, que le confirió un aspecto más opaco [29]. La biopelícula con el menor valor de opacidad, y por ende la más transparente, se consiguió con el T1.

Por otro lado, el valor de transparencia (VT), determinado a los 14 días después de su obtención, varió entre 1,36 y 8,29 $\mathrm{A}_{600} / \mathrm{mm}$ (Tabla 3 ), siendo T2 el que obtuvo el menor VT. Estos niveles estuvieron dentro de los rangos reportados para biopelículas con CS y aceite de germen de maíz [29] y con proteína de soja [41]. El comportamiento del VT fue similar a las demás propiedades ópticas (blancura, opacidad y $\Delta E^{*}$ ), que se vieron influenciados por la incorporación de solutos en la formulación, como el Sb y MC.

De manera general, las biopelículas del presente estudio fueron más opacas que las preparadas con CS y AE de canela y jengibre libres [42], proteínas de merluza [33], proteína de trigo [43] y proteína de soya [44]. Estas diferencias podrían deberse al tipo de proteína, origen y forma de incorporación del AE, que influyen en las propiedades ópticas de las biopelículas, particularmente en la transparencia y color $[43,45]$. En el presente estudio, se observó que las biopelículas con menores niveles de relación CS:Sb y CS:MC, mostraron las mejores propiedades ópticas.

\section{Propiedades mecánicas}

Las propiedades mecánicas se consideran uno de los atributos más importantes de los materiales de envasado de alimentos. La capacidad máxima de las películas para resistir el esfuerzo aplicado se mide por la (RT), mientras que el porcentaje de alargamiento en la rotura $\left(\varepsilon_{B}\right)$, es una propiedad mecánica que proporciona información sobre la deformación de un material antes de la rotura. Estas propiedades son importantes en el material de embalaje para proteger los alimentos envasados del deterioro debido a daños mecánicos y para mantener su integridad durante el almacenamiento de los procesos logísticos [46]. Las propiedades mecánicas de las biopelículas activas se resumen en la Tabla 4.

El $\varepsilon_{\mathrm{B}}$ varió entre 0,55 y $62,97 \%$. Al evaluar el efecto de los niveles dentro de cada factor, se observó que el incremento de $\mathrm{Sb}$ en la formulación también aumentó el porcentaje $\%$ de $\varepsilon_{B}$, por el contrario el incremento de MC en la formulación, redujo este parámetro. Los tratamientos con mayor porcentaje de deformación fueron T4 y T7, donde se notó que los mayores niveles del plastificante y menores niveles de MC, favorecieron el aumento de la deformación. Estos resultados son similares a los reportados para biopelículas con CS, glicerol y carvacrol $[14,15]$, CS, glicerol y AE de germen de maíz [29], en los que se observaron relaciones directas entre los niveles de plastificante y $\varepsilon B$; además, presentaron una reducción del $\varepsilon B$ con la incorporación del AE.

El módulo elástico $(E)$ varió entre 52,7 y 493 $\mathrm{MPa}$. El aumento de sorbitol en la formulación redujo el $E$; por el contrario, el incremento de MC lo elevó. Al analizar la

Tabla 4. Propiedades mecánicas de las biopelículas activas en todos los tratamientos evaluados.

\begin{tabular}{|c|c|c|c|c|c|c|c|c|c|}
\hline \multirow{3}{*}{$\begin{array}{l}\text { Tratamiento (T) } \\
\text { T1: } 0,5+0,5\end{array}$} & \multicolumn{9}{|c|}{ Propiedades mecánicas } \\
\hline & \multicolumn{3}{|c|}{$\begin{array}{c}\text { Alargamiento a la rotura } \\
-\boldsymbol{\varepsilon}^{\mathbf{B}}(\mathbf{\%}) \\
\mathrm{X} \pm \mathrm{DE}\end{array}$} & \multicolumn{3}{|c|}{$\begin{array}{l}\text { Módulo elástico - E (MPa) } \\
\qquad X \pm D E\end{array}$} & \multicolumn{3}{|c|}{$\begin{array}{c}\text { Resistencia a la tracción - } \\
\text { RT (MPa) } \\
\mathrm{X} \pm \mathrm{DE}\end{array}$} \\
\hline & $41,22^{1}$ & \pm & $4,18 \mathrm{~b}$ & 239,47 & \pm & $24,56 \mathrm{c}$ & 8,42 & \pm & $0,96 \mathrm{e}$ \\
\hline $\mathrm{T} 2: 0,5+1,0$ & 2,42 & \pm & $0,41 \mathrm{e}$ & 407,46 & \pm & $42,19 \mathrm{~d}$ & 5,65 & \pm & $1,10 \mathrm{~d}$ \\
\hline T3: $0,5+1,5$ & 0,55 & \pm & $0,13 \mathrm{e}$ & 492,96 & \pm & $62,49 \mathrm{e}$ & 4,43 & \pm & $1,14 \mathrm{bcd}$ \\
\hline T4: $1,0+0,5$ & 62,97 & \pm & $11,23 \mathrm{a}$ & 72,91 & \pm & $7,09 \mathrm{a}$ & 5,99 & \pm & $0,72 \mathrm{~d}$ \\
\hline T5: $1,0+1,0$ & 33,50 & \pm & $2,14 b c$ & 100,24 & \pm & $8,50 \mathrm{ab}$ & 4,64 & \pm & $0,22 \mathrm{bcd}$ \\
\hline T6: $1,0+1,5$ & 15,58 & \pm & $1,45 \mathrm{de}$ & 163,24 & \pm & $12,05 \mathrm{bc}$ & 4,85 & \pm & $0,58 \mathrm{bcd}$ \\
\hline $\mathrm{T} 7: 1,5+0,5$ & 61,14 & \pm & $9,91 \mathrm{a}$ & 52,72 & \pm & $2,85 \mathrm{a}$ & 2,88 & \pm & $0,08 \mathrm{abc}$ \\
\hline T8: $1,5+1,0$ & 39,09 & \pm & $5,96 \mathrm{~b}$ & 55,32 & \pm & $2,57 \mathrm{a}$ & 1,90 & \pm & $0,18 \mathrm{a}$ \\
\hline T9: $1,5+1,5$ & 22,70 & \pm & $1,06 \mathrm{~cd}$ & 68,04 & \pm & $4,32 \mathrm{a}$ & 2,76 & \pm & $0,23 \mathrm{ab}$ \\
\hline
\end{tabular}

${ }^{1}$ Los valores indican promedio ( $\mathrm{n}=3$ ). X: promedio; DE: desviación estándar. Letras diferentes, en sentido vertical, indican diferencias estadísticamente significativa según la prueba Tukey $(\mathrm{p}<0,05)$, para cada tratamiento $(\mathrm{T})$. 
interacción de los factores, se observó que los tratamientos T4, T7, T8 y T9, en su mayoría con mayor contenido de plastificante, mostraron menores valores de $E$. Por su lado, la (RT) varió entre 1,9 y 8,4 MPa. Se observó el aumento de los niveles de $\mathrm{Sb}$ y MC redujeron significativamente la RT. Los tratamientos con menores valores de RT fueron T7, T8 y T9; todos con mayor contenido de sorbitol. Tanto $E$ como RT mostraron comportamientos similares, notándose que el plastificante sorbitol es el principal responsable de la reducción de ambas propiedades mecánicas, las mismas que se ven afectadas, cuando se incorporan otros solutos, como AA libres o microencapsulados. Estos resultados fueron similares a los indicados por Arrieta et al. [14] y Akhter et al. [46] para biopelículas de CS, glicerol y AA.

Por otro lado, estudios previos reportan que los AA, como citral, carvacrol, timol y otros, afectan de alguna manera las interacciones entre las cadenas macromoleculares en la matriz del polímero. Este efecto puede estar relacionado con interacciones electrostáticas entre el CS y el AA, debido a las diferentes distribuciones de carga en las cadenas de proteína. Se puede afirmar que los caseinatos actúan como macroaniones en el $\mathrm{pH}$ experimental $(6,3-6,6)$; mientras que los AA, como el citral (por ser un aldehído acíclico con grupo funcionalCHO), podría ser portador de protones, intercambiando su protón por otro catión, como el sodio con carga positiva [15]. Otra manera de cómo la incorporación del AA afecta las propiedades mecánicas de las biopelículas, podría atribuirse a que los AA de tipo lipofílicos incrustados en la matriz de CS, pueden actuar como partículas de relleno deformables y mejorar las propiedades de tracción de las biopelículas [29]. Los efectos positivos de los AA en la elasticidad de las biopelículas son mayores cuando se incorpora en forma libre, pero se ven significativamente reducidos cuando se adiciona microencapsulado [16].

La mayoría de las investigaciones donde se evaluaron CS, plastificantes y AA de tipo lipofílico, coinciden en que las propiedades mecánicas de las biopelículas son influenciadas positivamente por la incorporación de plastificante; las películas no plastificadas presentan un $E$ y RT elevados a expensas de un $\varepsilon B$ reducido; comportamiento opuesto $(E$ y RT inferior, y mayor $\varepsilon \mathrm{B}$ ) se registra para muestras con glicerol $[15,29,30,47]$ y sorbitol [48], lo que confirma el papel del plastificante.

En términos generales, se sabe que las películas para envase de alimentos requieren una gran flexibilidad a temperatura ambiente para evitar roturas innecesarias durante su uso [49]. En tal sentido, se demostró que las biopelículas del presente estudio, con alto nivel de relación $\mathrm{CS}: \mathrm{Sb}$ y bajo nivel de CS:MC, tuvieron respuestas mecánicas adecuadas para envasado de alimentos. De los valores obtenidos para las propiedades ópticas y mecánica, se puede decir que el tratamiento que mostró resultados adecuados estuvo conformado por un nivel medio de CS:Sb $(1: 1,0)$ y menor nivel de CS:MC $(1: 0,5)$, cuya imagen fotográfica de la biopelícula se muestra en la Figura 1.

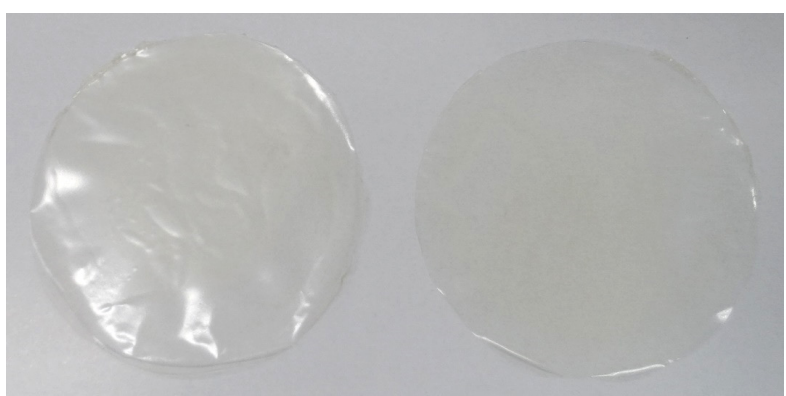

Figura 1. Fotografía de las biopelículas activas con micropartículas de citral elaboradas con relaciones $\mathrm{CS}: \mathrm{Sb}$ de $(1: 1,0)$ y CS:MC de $(1: 0,5)$; tomadas inmediatamente después de su procesamiento.

\section{Conclusiones}

Los niveles evaluados de CS:Sb y CS: MC influyeron significativamente en todas las variables analizadas. El incremento de los niveles de CS:Sb y CS:MC aumentó significativamente el espesor e influyó negativamente en las propiedades ópticas de las biopelículas. En cuanto a las propiedades mecánicas, las biopelículas activas mostraron una mayor elasticidad con el aumento progresivo de Sb, por el contrario, se vieron afectadas con el incremento de MC. Por lo tanto, si se considera la obtención de biopelículas con las mejores propiedades mecánicas (mayor $\boldsymbol{\varepsilon}_{\mathrm{B}} \%$, menor RT y menor E), sin afectar su espesor y propiedades ópticas, entre todas las formulaciones probadas, los resultados sobresalientes se encontraron para biopelículas con nivel medio de CS:Sb $(1: 1,0)$ y menor nivel de CS:MC $(1: 0,5)$. Esta formulación podría asegurar las condiciones para el procesamiento de la biopelícula, así como, la presencia significativa del antimicrobiano (MC), para obtener un sistema de envasado activo. En consecuencia, estas biopelículas muestran potencial para su uso futuro en la conservación de alimentos frescos. Finalmente, son necesarios más estudios sobre las propiedades funcionales relacionadas con los materiales en contacto con alimentos, es decir, microestructura, propiedades antimicrobianas, biodegradabilidad, permeabilidad al oxígeno y vapor de agua.

\section{Agradecimientos}

El primer autor agradece el apoyo al Consejo Nacional de Ciencia y Tecnología (CONCYTEC) por financiar la presente investigación y otorgar la beca para cursar el doctorado en Ciencia de Alimentos, dentro del programa doctoral de la Universidad Nacional Agraria La Molina - Lima, bajo el convenio de subvención 179-2015-FONDECYT. 


\section{Referencias bibliográficas}

[1] García C.J.: “Conozca las oportunidades para el sector envases y embalajes en Latinoamérica”. https:// www.camaralima.org.pe/repositorioaps/0/0/par/ r827_3/comercio exterior.pdf. (2018). (Consulta realizada: Diciembre, 2019)

[2] Aguirre-Joya J.A., Leon-Zapata M.A.D., Alvarez-Perez O.B., Torres-León C., Nieto-Oropeza D.E., VenturaSobrevilla J.M., Aguilar, M.A., Ruelas-Chacón, X., Rojas, R., Ramos-Aguiñaga, M.E. and Aguilar, C.N.: "Chapter 1 - Basic and applied concepts of edible packaging for foods". In: A.M. Grumezescu and A.M. Holban (eds). Food Packaging and Preservation. Elsevier Inc., Texas, USA, (2018), 1-61.

[3] Kim Y.T., Min B. and Kim K.W.: “Chapter 2 - General Characteristics of Packaging Materials for Food System". In: J.H. Han (ed). Innovations in Food Packaging. 2th. ed. Elsevier Ltd, Texas, USA, (2014), 13-35.

[4] Byun Y., Zhang Y. and Geng X.: "Chapter 5 Plasticization and Polymer Morphology". In: J.H. Han (ed). Innovations in Food Packaging. 2th. ed. Elsevier Ltd., Texas, USA, (2014), 87-108.

[5] Bucci D.Z., Tavares L.B.B. and Sell I.: "PHB packaging for the storage of food products". Polym. Test., Vol. 24, No. 5 (2005), 564-571.

[6] MINAM.: "Menos plástico más vida: Cifras del mundo y el Perú". Ministerio del Ambiente-MINAM. http://www.minam.gob.pe/menos-plastico-masvida/cifras-del-mundo-y-el-peru (2019) [Consulta realizada: enero 2019].

[7] Sengupta T. and Han JH.: "Chapter 4 - Surface Chemistry of Food, Packaging, and Biopolymer Materials". In: J.H. Han (ed). Innovations in Food Packaging. 2th. ed. Elsevier Ltd., Texas, USA, (2014), 51-86.

[8] Salgado P.R., Ortiz C.M., Musso Y.S., Giorgio L.D. and Mauri A.N. "Edible films and coatings containing bioactives". Food Sci., Vol. 5 (2015), 86-92.

[9] Falguera V., Quintero J.P., Jiménez A., Muñoz J.A. and Ibarz A.: "Edible films and coatings: Structures, active functions and trends in their use". Trends Food Sci. Technol., Vol. 22, No. 6 (2011), 292-303.

[10]. Menezes J. and Athmaselvi K.A.: "Chapter 5 - Report on edible films and coatings". In: A.M. Grumezescu and A.M. Holban (eds). Food Packaging and Preservation. Elsevier Inc., Texas USA, (2018), 177212.

[11] Pereda M., Aranguren M.I. and Marcovich N.E.: "Characterization of Chitosan/Caseinate Films". J. Appl. Polym. Sci., Vol. 107 (2008), 1080-1090.

[12] Barreto P.L.M., Pires A.T.N. and Soldi V.: "Thermal degradation of edible films based on milk proteins and gelatin in inert atmosphere". Polym. Degrad. Stab., Vol. 79, No. 1 (2003), 147-152.

[13] Audic J.L., Chaufer B. and Daufin G.: "Non-food applications of milk components and dairy coproducts: A review". Lait, Vol. 83 (2003), 417-438.

[14] Arrieta M.P., Peltzer M.A., López J., Garrigós M.D.C., Valente A.J.M. and Jiménez A.: "Functional properties of sodium and calcium caseinate antimicrobial active films containing carvacrol". J. Food Eng., Vol. 121, No. 1 (2014), 94-101.

[15] Arrieta M.P., Peltzer M.A., Garrigós M.D.C. and Jiménez A.: "Structure and mechanical properties of sodium and calcium caseinate edible active films with carvacrol". J. Food Eng., Vol. 114, No. 4 (2013), 486-494.

[16] Alarcón-Moyano J.K., Bustos R.O., Herrera M.L. and Matiacevich S.B.: "Alginate edible films containing microencapsulated lemongrass oil or citral: effect of encapsulating agent and storage time on physical and antimicrobial properties". J. Food Sci. Technol., Vol. 54, No. 9 (2017), 2878-1889.

[17] Bustos C.R.O., Alberti F.V. and Matiacevich S.B.: "Edible antimicrobial films based on microencapsulated lemongrass oil". J. Food Sci. Technol., Vol. 53, No. 1 (2016), 832-839.

[18] Atarés L. and Chiralt A.: "Essential oils as additives in biodegradable films and coatings for active food packaging". Trends Food Sci. Technol., Vol. 48 (2016), 51-62.

[19] Corrales M., Fernández A. and Han J.H.: "Chapter 7 Antimicrobial Packaging Systems”. In: J.H. Han (ed). Innovations in Food Packaging. 2th. ed. Elsevier Ltd., Texas, USA, (2014), 133-170.

[20] Lee D.S.: "Chapter 6 - Antioxidative Packaging System". In: J.H. Han (ed). Innovations in Food Packaging. 2th. ed. Elsevier Ltd., Texas, USA, (2014). 111-131.

[21] Ribeiro-Santos R., Andrade M. and Sanches-Silva A.: "Application of encapsulated essential oils as antimicrobial agents in food packaging". Curr. Opin Food Sci., Vol. 14 (2017), 78-84.

[22] Stashenko E.E.: “Aceites Esenciales". División de Publicaciones UIS. (2009), 180.

[23] Bonilla J., Poloni T., Lourenço R.V. and Sobral P.J.A.: "Antioxidant potential of eugenol and ginger essential oils with gelatin/chitosan films". Food Biosci., Vol. 23 (2018), 107-114.

[24] Caetano K. dos S., Lopes N.A., Costa T.M.H., Brandelli A., Rodrigues E., Flôres S.H. and Cladera-Olivera, F.: "Characterization of active biodegradable films based on cassava starch and natural compounds". Food Packag. Shelf Life, Vol. 16 (2018), 138-147.

[25] Navikaite-snipaitiene V., Ivanauskas L., Jakstas V., 
Rüegg N., Rutkaite R., Wolfram E. and Yildirim, S.: "Development of antioxidant food packaging materials containing eugenol for extending display life of fresh beef". Meat Sci., Vol. 145 (2018), 9-15.

[26] López P., Gándara J. and Losada P.: “Infrared spectrophotometric determination of citral corrected for limonene interference in lemon and orange essential oils". Food Chem., Vol. 46, No. 2, (1993), 193-197.

[27] Maswal M. and Dar A.A.: "Formulation challenges in encapsulation and delivery of citral for improved food quality". Food Hydrocoll., Vol. 37 (2014), 182195.

[28] Saddiq A.A. and Khayyat S.A.: "Chemical and antimicrobial studies of monoterpene: Citral. Pestic". Biochem Physiol., Vol. 98, No. 1 (2010), 89-93.

[29] Matsakidou A., Tsimidou M.Z. and Kiosseoglou V.: "Storage behavior of caseinate-based films incorporating maize germ oil bodies". Food Res. Int., Vol. 116 (2019), 1031-1040.

[30] Pereda M., Aranguren M.I. and Marcovich N.E.: "Caseinate films modified with tung oil". Food Hydrocoll., Vol. 24, No. 8 (2010), 800-808.

[31] Yoplac I., Avila-George H., Vargas L., Robert P. and Castro W.: "Determination of the superficial citral content on microparticles: An application of NIR spectroscopy coupled with chemometric tools". Heliyon, Vol. 5 (2019), e02122.

[32] Yoplac I., Avila-George H., Vargas L., Robert P. and Castro W. "Prediction of citral superficial content on microparticles by NIR spectroscopy and partial least square regression". Rev. Técnica Ing. la Univ. Zulia, Vol. 42, No. 2 (2019), 76-85.

[33] Pires C., Ramos C., Teixeira G., Batista I., Mendes R., Nunes L. and Marques, A.: "Characterization of biodegradable films prepared with hake proteins and thyme oil". J. Food Eng., Vol. 105, No. 3 (2011), 422-428.

[34] HunterLab Method.: "Applications Note: Opacity" https: / / www.hunterlab.se / wp-content / uploads/2012/11/0pacity.pdf.(2008) (Consultation made: Julio 2019).

[35] Alonso J.V.: "Taller sobre el color y su medición". http: / / oa.upm.es / 42855 / 1 / Taller_color_ JVAlonsoFelipe.pdf. (2016). (Consulta realizada: Agosto 2019).

[36] Wold J.H. and Valberg A.: "The derivation of XYZ tristimulus spaces: A comparison of two alternative methods". Color Res Appl., Vol. 26, No. S1 (2001), S222-S224.

[37] Hamaguchi P.Y., WuYin W. and Tanaka M.: "Effect of $\mathrm{pH}$ on the formation of edible films made from the muscle proteins of Blue marlin (Makaira mazara)". Food Chem., Vol. 100, No. 3 (2007), 914-920.
[38] Shiku Y., Hamaguchi P.Y. and Tanaka M.: "Effect of $\mathrm{pH}$ on the preparation of edible films based on fish myofibrillar proteins". Fish Sci., Vol. 69 (2003), 1026-1032.

[39] ASTM.: "Standard Test Method for Tensile Properties of Thin Plastic Sheeting". Standards Designation: D882-01 Annual Book of ASTM Standards ASTM Philadelphia, USA. (2001), 10.

[40] García F.T. and Sobral P.J.: "Effect of the thermal treatment of the filmogenic solution on the mechanical properties, color and opacity of films based on muscle proteins of two varieties of Tilapia". LWT - Food Sci. Technol., Vol. 38 (2005), 289-296.

[41] Ciannamea E.M., Stefani P.M. and Ruseckaite R.A.: "Storage-induced changes in functional properties of glycerol plasticized e Soybean protein concentrate fi lms produced by casting". Food Hydrocoll., Vol. 45 (2015), 247-255.

[42] Atarés L., Bonilla J. and Chiralt A.: “Characterization of sodium caseinate-based edible films incorporated with cinnamon or ginger essential oils". J. Food Eng., Vol. 100, No. 4 (2010), 678-687.

[43] Irissin-mangata J., Bauduin G., Boutevin B. and Gontard N.: "New plasticizers for wheat gluten films". Eur. Polym J., Vol. 37 (2001), 1533-1541.

[44] Jensen A., Lim L.T., Barbut S. and Marcone M.: "Development and characterization of soy protein films incorporated with cellulose fibers using a hot surface casting technique". LWT - Food Sci. Technol., Vol. 60, No. 1 (2015), 162-170.

[45] Quintavalla S. and Vicini L.: "Antimicrobial food packaging in meat industry". Meat Sci., Vol. 62 (2002), 373-380.

[46] Akhter R, Masoodi F.A., Wani T.A. and Rather S.A.: "Functional characterization of biopolymer based composite film: Incorporation of natural essential oils and antimicrobial agents". Int. J. Biol. Macromol., Vol. 137 (2019), 1245-1255.

[47] Montero P., Mosquera M., Marín-peñalver D., Alemán A., Martínez-álvarez Ó. And Gómez-guillén M.C.: "Changes in structural integrity of sodium caseinate fi lms by the addition of nanoliposomes encapsulating an active shrimp peptide fraction". J. Food Eng., Vol. 244 (2019), 47-54.

[48] Tapia-Blácido D.R., do Amaral Sobral P.J. and Menegalli F.C.: "Optimization of amaranth flour films plasticized with glycerol and sorbitol by multiresponse analysis". LWT - Food Sci. Technol., Vol. 44, No. 8 (2011), 1731-1738.

[49] Martino V.P., Ruseckaite R.A. and Jiménez A.: "Thermal and mechanical characterization of plasticized poly (L-lactide-co-D,L-lactide) films for food packaging". J. Therm. Anal. Calorim., Vol. 3 (2006), 707-712. 


\section{REVISTA TECNICA}

DE LA FACULTAD DE INGENIERIA

UNIVERSIDAD DEL ZULIA

Vol. 44. N`1, Enero - Abril 2021, pp. 04 - 58

Esta revista fue editada en formato digital y publicada en Diciembre de 2020, por el Fondo Editorial Serbiluz, Universidad del Zulia. Maracaibo-Venezuela

www.luz.edu.ve

www.serbi.luz.edu.ve

www.produccioncientificaluz.org 